\title{
Tools for Dark Matter Indirect Detection
}

\author{
Marco Cirelli*十 \\ Laboratoire de Physique Théorique et Hautes Energies (LPTHE), \\ UMR 7589 CNRS \& UPMC, 4 Place Jussieu, F-75252, Paris, France \\ E-mail: marco.cirelli@gmail.com
}

I discuss ingredients and recipes for computing signals of TeV-scale Dark Matter annihilations and decays in the Galaxy and beyond: the energy spectra of electrons and positrons, antiprotons, antideuterons, gamma rays, neutrinos and antineutrinos e, mu, tau at production, the propagation functions for charged particles in the Galaxy, the energy spectra at the location of the Earth, the gamma ray fluxes from the Galaxy and from beyond. All results discussed here are available in numerical form and ready to be consumed.

Corfu Summer Institute 2017 'School and Workshops on Elementary Particle Physics and Gravity' 2-28 September 2017

Corfu, Greece

\footnotetext{
${ }^{*}$ Speaker.

$\dagger$ This work has been done in part within the Labex ILP (reference ANR-10-LABX-63) part of the Idex SUPER, and received financial state aid managed by the Agence Nationale de la Recherche, as part of the programme Investissements d'avenir under the reference ANR-11- IDEX-0004-02. The work of M.C. is supported by the European Research Council (ERC) under the EU Seventh Framework Programme (FP7/2007-2013) / ERC Starting Grant (agreement $n$. 278234 - 'NEWDARK' project). M.C. acknowledges the hospitality of the Institut d'Astrophysique de Paris (IAP) where a part of this work was done.
} 


\section{Introduction}

Cosmology and astrophysics provide several convincing evidences of the existence of Dark Matter (DM). The observation that some mass is missing to explain the internal dynamics of galaxy clusters and the rotations of galaxies dates back respectively to the '30s and the '70s. The observations from weak lensing, for instance in the spectacular case of the so-called 'bullet cluster', provide evidence that there is mass where nothing is optically seen. More generally, global fits to a number of cosmological datasets (Cosmic Microwave Background, Large Scale Structure and also Type Ia Supernovae) allow to determine very precisely the amount of DM in the global energymatter content of the Universe at $\Omega_{\mathrm{DM}} h^{2}=0.1123 \pm 0.0035$.

All these signals pertain to the gravitational effects of Dark Matter at the cosmological and extragalactical scale. Searches for explicit manifestation of the DM particles that are supposed to constitute the halo of our own galaxy (and the large scale structures beyond it) have instead so far been giving negative results, but this might be on the point of changing.

Indirect searches for Dark Matter aim at detecting the signatures of the annihilations or decays of DM particles in the fluxes of Cosmic Rays (CRs), intended in a broad sense: charged particles (electrons and positrons, protons and antiprotons, deuterium and antideuterium), photons (gamma rays, $\mathrm{X}$-rays, synchrotron radiation), neutrinos. In general, a key point of all these searches is to look for channels and ranges of energy where it is possible to beat the background from ordinary astrophysical processes. This is for instance the basic reason why searches for charged particles focus on fluxes of antiparticles (positrons, antiprotons, antideuterons), much less abundant in the Universe than the corresponding particles, and searches for photons or neutrinos have to look at areas where the DM-signal to astro-noise ratio can be maximized.

Pioneering works have explored indirect detection (ID) as a promising avenue of discovery since the late-70's. Since then, innumerable papers have explored the predicted signatures of countless particle physics DM models. In the past 9 years or so, however, the field has experienced a significant burst of activity, mainly due to the results presented by a few very well performing experiments, above all the PAMELA satellite, the AMS experiment, the FERMI satellite, the HESS telescope and others. It is fair to say that the field has passed, for better or for worse, from a theory-driven state to a data-driven phase.

The next few years promise to be even richer of data from Dark Matter Indirect Searches (certainly in terms of further explorations of the parameter spaces, and hopefully even with a positive detection). It will therefore be useful to have at disposal a set of consistent tools that can allow to interpret these data in a model independent way and cross-check between different channels. This is what we have aimed at realizing in [1] and that I am going to briefly present in this text. While the complete discussions can be found in [1], here I will focus only on some aspects and on the general infrastructure, leaving most of the formulae outside. All the results are available in numerical form in [2].

\section{Fluxes at production}

The first ingredients that one needs to compute DM ID signatures are of course the fluxes of stable Standard Model particles $\left(e^{ \pm}, \bar{p}, \bar{d}, \gamma, \stackrel{(-)}{v}_{e, \mu, \tau}\right)$ originating from DM annihilations, or decays. 

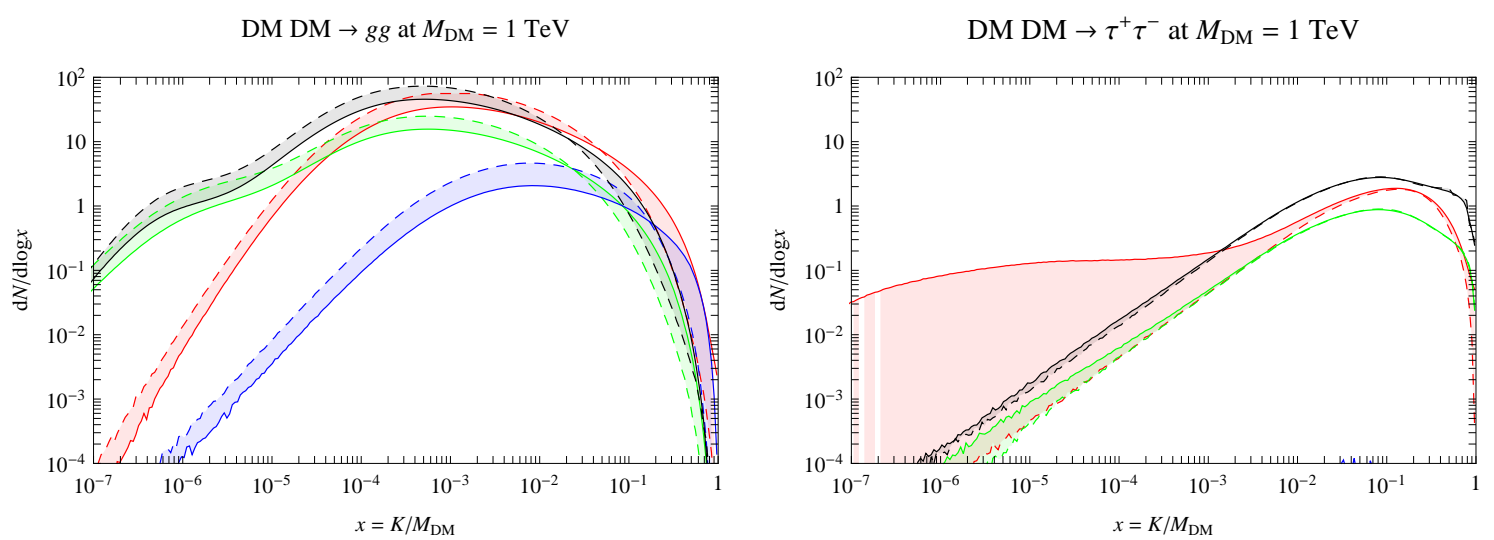

Figure 1: Comparison between Monte Carlo results: Pythia is the continuous line, HeRwiG is dashed. Photons (red), $e^{ \pm}$(green), $\bar{p}$ (blue), $v=v_{e}+v_{\mu}+v_{\tau}$ (black).

We want to be as model independent as possible and so we consider DM annihilations (parameterized by the DM DM cross section $\sigma v$ ) and decays (described by the DM decay rate $\Gamma=1 / \tau$ ) into a large number of primary channels:

$$
\begin{aligned}
& e_{L}^{+} e_{L}^{-}, e_{R}^{+} e_{R}^{-}, \mu_{L}^{+} \mu_{L}^{-}, \mu_{R}^{+} \mu_{R}^{-}, \tau_{L}^{+} \tau_{L}^{-}, \tau_{R}^{+} \tau_{R}^{-}, q \bar{q}, c \bar{c}, b \bar{b}, t \bar{t}, \gamma \gamma, g g, \\
& W_{L}^{+} W_{L}^{-}, W_{T}^{+} W_{T}^{-}, Z_{L} Z_{L}, Z_{T} Z_{T}, h h \\
& v_{e} \bar{v}_{e}, v_{\mu} \bar{v}_{\mu}, v_{\tau} \bar{v}_{\tau}, V V \rightarrow 4 e, V V \rightarrow 4 \mu, V V \rightarrow 4 \tau,
\end{aligned}
$$

where $q=u, d, s$ denotes a light quark and $h$ is the Standard Model (SM) Higgs boson. The last three channels denote models in which the annihilation or decay first happens into some new (light) boson $V$ which then decays into a pair of leptons, along the lines of the models proposed in [3,4].

All the spectra are available on [2]. Here I want to discuss two general issues: i) the intrinsic uncertainty in computing those spectra with numerical methods and ii) the impact of ElectroWeak radiation.

\subsection{MonteCarlo 'uncertainty'}

Almost every DM indirect search analysis uses the collider MonteCarlo code PYTHIA to compute the annihilation spectra, despite the fact that other codes are available and that in any case all codes have been designed and calibrated for the collider environment and in an energy range which (until recently) was much lower than the multi-TeV one of interest for some DM models.

In order to get a feeling of the intrinsic uncertainty related to these issues, in [1] we employed the two most widely used MonteCarlo simulation programs: Pythia [5] (version 8.135) and HeRWIG [6] (version 6.510). In fact, the algorithms implemented in HERWIG and PyTHIA are quite different, in both parton showers and hadronization.

The discrepancies can be tentatively quoted at $\pm 20 \%$, although bigger surprises are possible for some channels. In particular, fig. 1 (right panel) shows a case in which the predictions from HeRwig largely underestimate the flux of photons at low energy. This is due to the fact that the latter does not include photon emission off leptons. 


\subsection{ElectroWeak radiation}

The emission of $W$ 's and $Z$ 's from the final (or initial!) states is enhanced by one or more powers of $\ln \left(M / M_{W, Z}\right)$, with $M \gg M_{W, Z}$, not depending on the DM model. For large $M$, these can be important and lead to significant modifications of the spectra [7]. First, the weak emission entails the presence of further unstable hadrons in the final state, and therefore it significantly modifies the flux of $\gamma^{\prime}$ s and $e^{ \pm}$at energies $E \ll M, M$ being the DM mass. Moreover, $W / Z$ radiation leads to a $\bar{p}$ contribution, even in annihilation channels that would be completely leptonic if EW radiation is neglected. For instance, a DM DM $\rightarrow v \bar{v}$ channel also yield $e^{ \pm}$'s, $\gamma$ 's and $\bar{p}$ 's, rather counterintuitively.

\section{Propagation of charged cosmic rays}

The $e^{-}, e^{+}$and $\bar{p}$ produced in any given point of the halo propagate immersed in the turbulent galactic magnetic field. The field consists of random inhomogeneities that act as scattering centers for charged particles, so that their journey can effectively be described as a diffusion process from an extended source (the DM halo) to some final given point (the location of the Earth, in the case of interest). While diffusing, charged CRs experience several other processes, and in particular energy losses due to synchrotron radiation, Inverse Compton Scattering (ICS) on the low energy photons of the CMB and starlight, Coulomb losses, bremsstrahlung, nuclear spallations... . Quantitatively, the steady-state number density $n_{f}(\vec{x}, E)$ per unit energy $E$ of the cosmic ray species $f\left(=e^{+}, e^{-}, \bar{p}\right)$ in any given point $\vec{x}$ obeys to a diffusion-loss equation [8]

$$
\begin{aligned}
-\mathscr{K}(E) \cdot \nabla^{2} n_{f}-\frac{\partial}{\partial E}\left(b(E, \vec{x}) n_{f}\right) & +\frac{\partial}{\partial z}\left(\operatorname{sign}(z) V_{\text {conv }} n_{f}\right) \\
& =Q(E, \vec{x})-2 h \delta(z) \Gamma n_{f} .
\end{aligned}
$$

The first term accounts for diffusion, with a coefficient conventionally parameterized as $\mathscr{K}(E)=$ $\mathscr{K}_{0}(E / \mathrm{GeV})^{\delta}$. The second term describes energy losses: the coefficient $b$ is position-dependent since the intensity of the magnetic field (which determines losses due to synchrotron radiation) and the distribution of the photon field (which determines losses due to ICS) vary across the galactic halo. It also has a dependence on energy which is equal to $E^{2}$ only as long as ICS is approximated with Thomson scattering. It is normalized by the value of the typical loss timescale at $1 \mathrm{GeV}$ at the location of the Earth $\tau_{\odot}=5.7 \times 10^{15} \mathrm{sec}$. This is illustrated in fig. 2 . The third term deals with convection while the last term accounts for nuclear spallations, that occur with rate $\Gamma$ in the disk of thickness $h \simeq 100 \mathrm{pc}$. The source, DM annihilations, is given by $Q=1 / 2(\rho(\vec{x}) / M)^{2} \sum_{i} \mathrm{BR}_{i}\langle\sigma v\rangle$ $\left(d N_{f}^{i} / d E\right)$, where $\sigma v$ is the total annihilation cross section and the sum runs over all primary channels $i$ in which the cosmic ray species $f$ is produced. $d N_{f}^{i} / d E$ ) are the spectra discussed in Sec. 2. $\rho(\vec{x})$ is the DM density distribution in the galactic halo. The different processes described above have a different importance depending on the particle species: the journey of electrons and positrons is primarily affected by synchrotron radiation and inverse Compton energy losses, while for antiprotons these losses are negligible and convection and spallation dominate.

Eq. (3.1) is usually solved numerically in a diffusive region with the shape of a solid flat cylinder that sandwiches the galactic plane, with height $2 L$ in the $z$ direction and radius $R=20 \mathrm{kpc}$ in the $r$ direction. The location of the solar system corresponds to $\vec{x}_{\odot}=\left(r_{\odot}, z_{\odot}\right)=(8.33 \mathrm{kpc}, 0)$. 

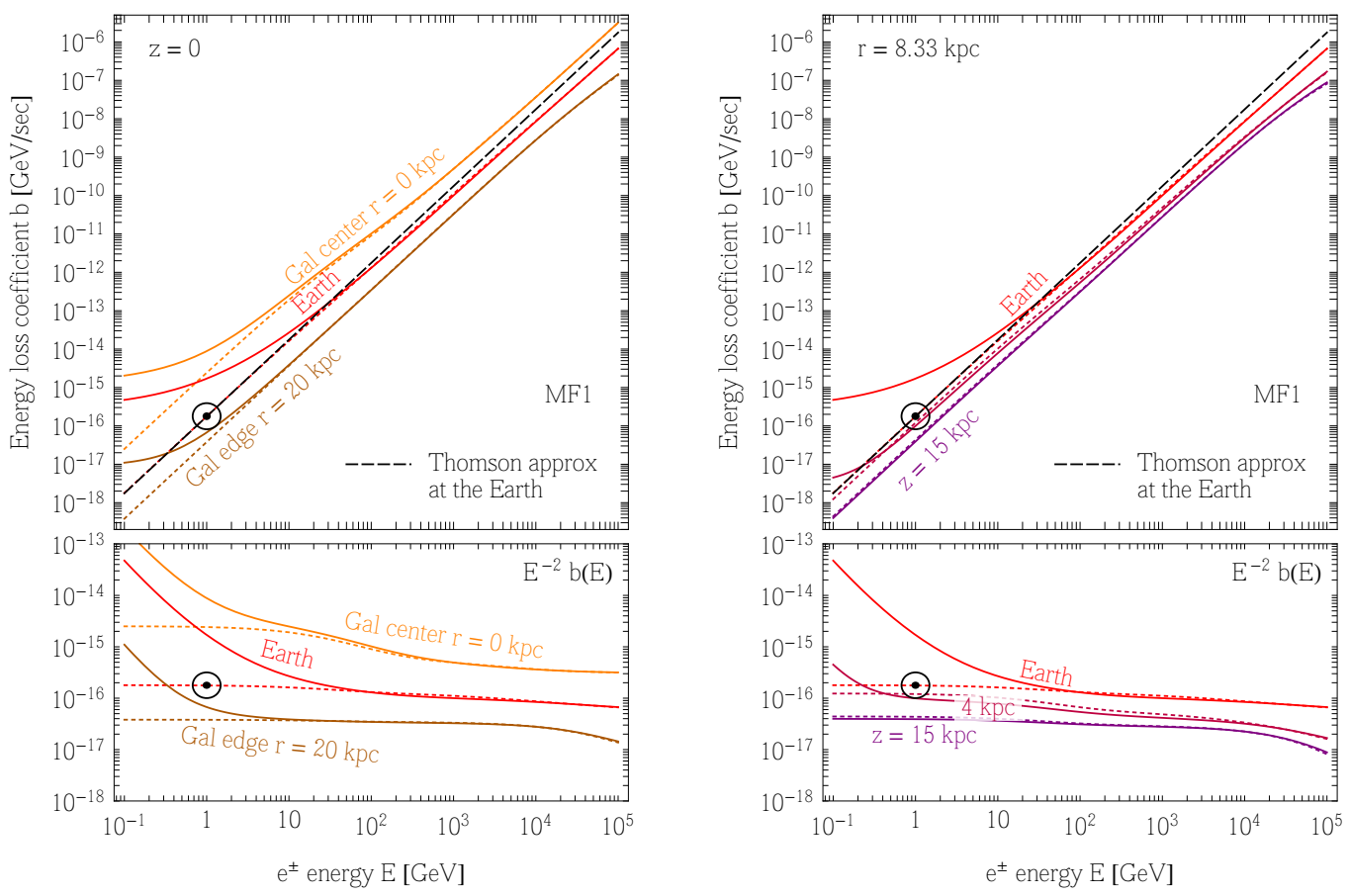

Figure 2: Energy loss coefficient function for electrons and positrons in the Milky Way. Left panel: at several locations along the galactic radial coordinate $r$, right panel: above (or below) the location of the Earth along the coordinate $z$. The dot points at the value of $\tau_{\odot}$.

Boundary conditions are imposed such that the number density $n_{f}$ vanishes on the surface of the cylinder, outside of which the charged cosmic rays freely propagate and escape. The values of the propagation parameters $\delta, K_{0}, V_{\text {conv }}$ and $L$ are deduced from a variety of (ordinary) cosmic ray data and modelizations.

In the usual solution scheme, the energy loss coefficient $b$ is considered as constant in space and proportional to $E^{2}$. In [1], we improved the numerical solution by including the correct space and energy dependence. This involves defining a set of energy-dependent 'halo functions' (see [1] for more details). Subsequently, in [9] we refined the procedure by notably including low energy losses.

Once the propagation is performed, one obtains the fluxes of charged cosmic rays at the location of the Earth. Depending on the choices for the propagation parameters, the resulting spectra can differ by more than one order of magnitude. Two representative examples can be found in fig. 3: positrons on the left and antiprotons on the right ${ }^{1}$. The MIN, MED, MAX labels refer indeed to different sets of propagation parameters. All numerical spectra are available in [2].

\section{Galactic $\gamma$ rays}

Dark Matter produces high energy gamma rays both by direct ('prompt') emission during

\footnotetext{
${ }^{1}$ The antiproton spectra have in turn be refined in [10].
} 

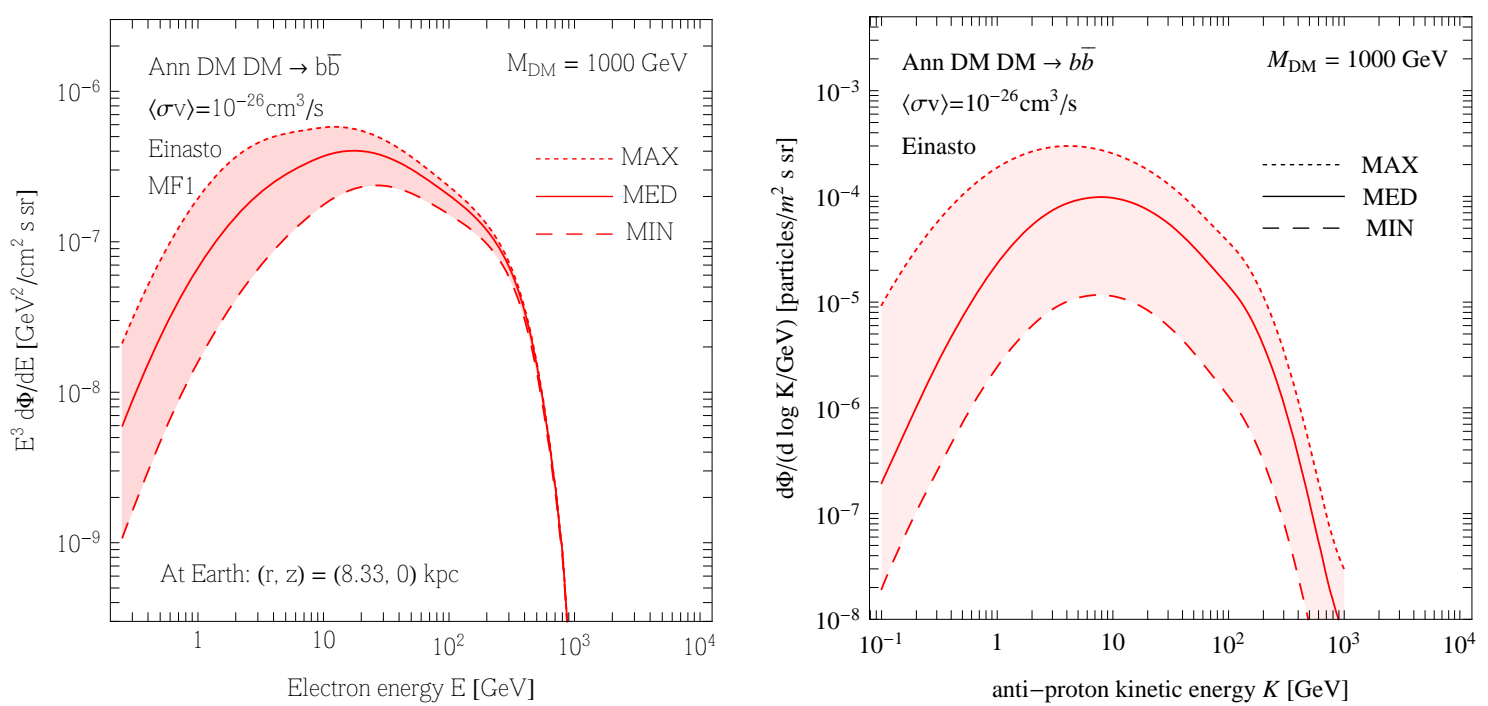

Figure 3: Examples of propagated fluxes of charged CRs at the location of the Earth. Positrons on the left and antiprotons on the right.

annihilation or decay and by Inverse Compton Scattering of $e^{ \pm}$produced by DM on the ambient light ('secondary'). The former are included in the fluxes discussed in Sec. 2. The latter have to be computed by folding the maps of propagated $e^{ \pm}$with the maps of target light, in particular in the Galaxy.

One of the best features of these fluxes of ICS $\gamma$ rays as possible signatures of DM is that they originate from 'everywhere' in the diffusion volume of the galactic halo, including regions where the astrophysical background is reduced (e.g. at high latitudes). Moreover, other energy losses (such as synchrotron radiation) are sub-dominant with respect to Inverse Compton energy losses essentially everywhere, so that, thanks to energy conservation, the resulting ICS $\gamma$ flux suffers only moderate astrophysical uncertainties.

In [1] (and then in [9]) we presented a compact formalism to compute galactic ICS and bremsstrahlung $\gamma$-ray fluxes, employing a set of functions similar to the halo functions that are introduced for the propagation of charged cosmic rays (briefly discussed above). An example of the resulting fluxes is reported in fig. 4, left. This example chooses a specific DM model and a small but not too small observational rectangular window of $5^{\circ}$ around the Galactic Center. The fluxes are plotted for different choices of DM profile, showing that variations of two orders of magnitude are possible. Again, all numerical spectra can be computed with the tools available in [2].

\section{Extragalactic $\gamma$ rays}

The $\gamma$-rays emitted by DM annihilations or decays in all the extragalactic structures and (in principle) all along the history of the Universe reach us in the form of an isotropic contribution to the total $\gamma$-ray intensity. In [1] we discuss in detail how to compute it. With respect to the galactic case, there are a few main differences: (i) one has to include the effect of the 'cosmological 

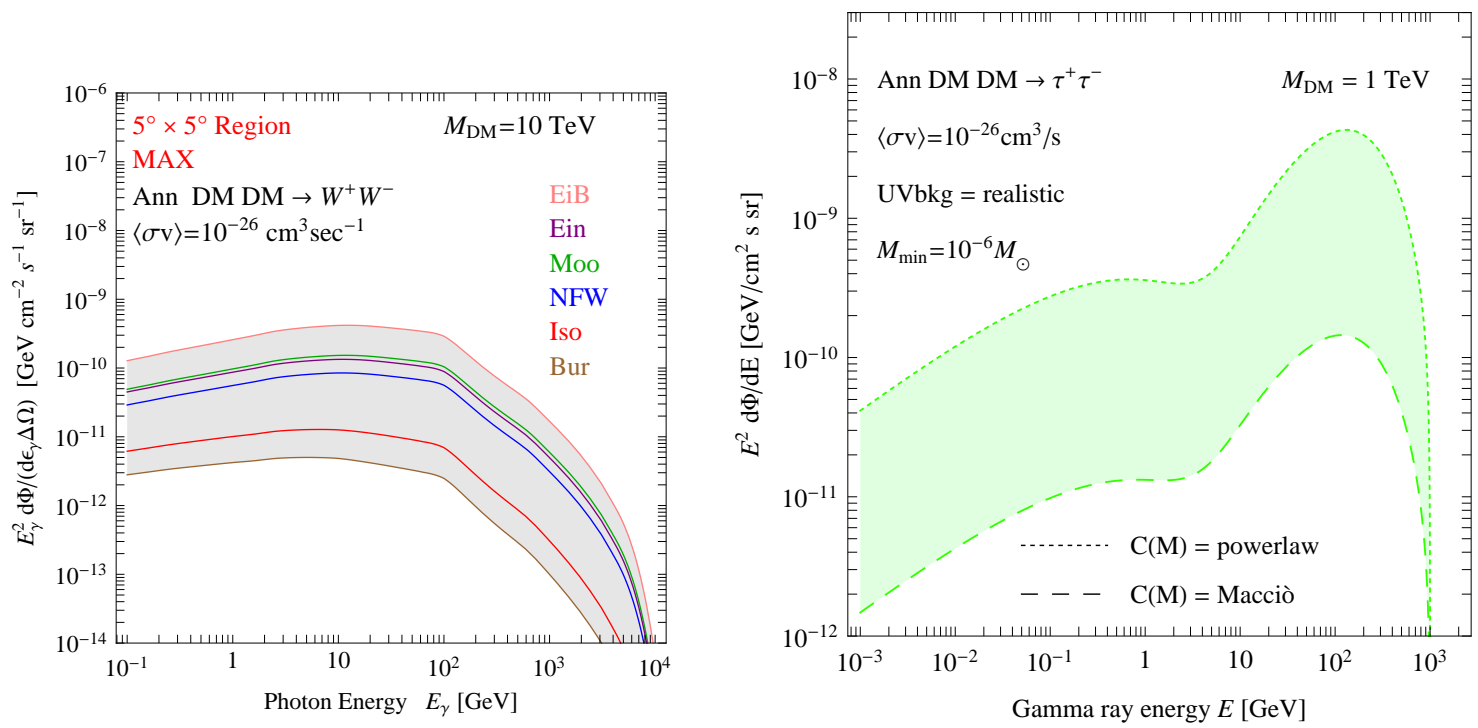

Figure 4: Left: example of fluxes of ICS $\gamma$-rays from the Galaxy, for different choices of the DM galactic profile. Right: example of fluxes of extragalactic $\gamma$-rays, illustrating the impact of changing the assumption for the concentration parameter.

dimming' due to the expansion of the Universe, (ii) one has to include the fact that, unlike in the galactic environment, on cosmologically large distances one can not neglect the absorption of gamma-rays and finally (iii) one has to account for the history of formation of DM structures. Fig. 4, right, shows an example of the resulting fluxes. One also sees that, varying the choice for the concentration function $C(M)$ (one of the parameters associated to the history of halo formation), the spread can be almost 2 orders of magnitude.

\section{Neutrinos}

Neutrinos are of course produced in DM annihilations together with all the other particles discussed above. Similarly to $\gamma$-rays, neutrinos have the advantage of proceeding straight and essentially unabsorbed through the Galaxy. Even more, they can cross long lengths of dense matter with little interaction. Contrary to $\gamma$-rays, however, the detection principle of neutrinos is more difficult and it introduces limitations in the choice of targets. Neutrinos are observed at huge Čerenkov detectors located underground (or under-ice or under-water) via the showers of secondary particles that they produce when interacting in the material inside the instrumented volume or in its immediate surroundings. The charged particles, in particular muons, emit Čerenkov light when traversing the experiment and thus their energy and direction (which are connected to those of the parent neutrino) can be measured. The main background for this search consists in the large flux of cosmic muons coming from the atmosphere above the detector. The experiments, therefore, have to select only upgoing tracks, i.e. due to neutrinos that have crossed the entire Earth and interacted inside or just below the instrumented volume. Schematically, experiments look for neutrinos: 
- From the Galactic Center (GC) or the Galactic Halo (GH), in close similarity with $\gamma$-rays. Experiments located at the South Pole can not directly 'see' the GC, which is essentially above horizon for them.

- From satellite galaxies or clusters of galaxies, again in similarity with $\gamma$-rays, although in this case the sensitivities are typically not competitive with gamma rays.

- From the center of the Sun (or even the Earth). The idea is that DM particles in the halo may become gravitationally captured by a massive body, lose energy via repeated scatterings with its nuclei and thus accumulate at its center. The annihilations occurring there give origin to fluxes of high energy neutrinos which, albeit suffering oscillations and interactions in the dense matter of the astrophysical body can emerge. The detection of high-energy neutrinos from the Sun, on top of the much lower energy neutrino flux due to nuclear fusion processes, would constitute the proverbial smoking gun for DM, as there are no known astrophysical processes able to mimic it.

The fluxes from the GC, the GH or satellite galaxies are included in the fluxes discussed in Sec. 2. The state of the art computational tools for neutrinos from the Sun are presented in [11].

\section{Conclusions}

The next few years will be rich of data in the field of indirect searches for Dark Matter. It will be interesting, from a phenomenological and model-independent point of view, to have at disposal all the tools needed to quickly interpret and cross check them. This is what we aimed at providing in [1], and the associated string of subsequent papers $[9,10,11]$. We make all numerical results downloadable from [2]. The main innovations introduced in these papers are: (i) a comparison between different MCs, (ii) an improved semi-analytical propagation for $e^{ \pm}$in the Galaxy, (iii) a set of 'halo functions' for computing ICS and bremsstrahlung $\gamma$-ray fluxes and synchrotron radio emission, (iv) a thorough analysis of the uncertainties that affect extragalactic $\gamma$-ray fluxes, (v) a state of the art computation of neutrinos from the Sun.

\section{References}

[1] M. Cirelli, G. Corcella, A. Hektor, G. Hutsi, M. Kadastik, P. Panci, M. Raidal and F. Sala et al., "PPPC 4 DM ID: A Poor Particle Physicist Cookbook for Dark Matter Indirect Detection," JCAP 1103 (2011) 051, arXiv:1012.4515 [hep-ph].

[2] www.marcocirelli.net/PPPC4DMID.html

[3] N. Arkani-Hamed, D. P. Finkbeiner, T. R. Slatyer, N. Weiner, Phys. Rev. D79 (2009) 015014, arXiv:0810.0713.

[4] M. Pospelov, A. Ritz, Phys. Lett. B671 (2009) 391-397, arXiv:0810.1502.

[5] T. Sjostrand, S. Mrenna and P.Z. Skands, Comput. Phys. Commun. 178 (2008) 852.

[6] G. Corcella et al., JHEP 0101 (2001) 010. 
[7] P. Ciafaloni, D. Comelli, A. Riotto, F. Sala, A. Strumia and A. Urbano, JCAP 1103 (2011) 019 doi:10.1088/1475-7516/2011/03/019 [arXiv:1009.0224 [hep-ph]].

P. Ciafaloni, M. Cirelli, D. Comelli, A. De Simone, A. Riotto and A. Urbano, JCAP 1106 (2011) 018 doi:10.1088/1475-7516/2011/06/018 [arXiv:1104.2996 [hep-ph]].

P. Ciafaloni, M. Cirelli, D. Comelli, A. De Simone, A. Riotto and A. Urbano, JCAP 1110 (2011) 034 doi:10.1088/1475-7516/2011/10/034 [arXiv:1107.4453 [hep-ph]].

[8] P. Salati, Indirect and direct dark matter detection, Proceedings of the 2007 Cargèse Summer School: Cosmology and Particle Physics Beyond the Standard Models, PoS(cargese)009, pos.sissa.it/cgi-bin/reader/conf.cgi?confid=49

[9] J. Buch, M. Cirelli, G. Giesen and M. Taoso, JCAP 1509 (2015) no.09, 037 doi:10.1088/1475-7516/2015/9/037, 10.1088/1475-7516/2015/09/037 [arXiv:1505.01049 [hep-ph]].

[10] M. Boudaud, M. Cirelli, G. Giesen and P. Salati, JCAP 1505 (2015) no.05, 013 doi:10.1088/1475-7516/2015/05/013 [arXiv:1412.5696 [astro-ph.HE]].

[11] P. Baratella, M. Cirelli, A. Hektor, J. Pata, M. Piibeleht and A. Strumia, JCAP 1403 (2014) 053 doi:10.1088/1475-7516/2014/03/053 [arXiv:1312.6408 [hep-ph]]. 